\title{
Folate hydrolase (prostate-specific antigen) 1 expression in bladder cancer subtypes and associated tumor neovasculature
}

\author{
Mary K Samplaski ${ }^{1}$, Warren Heston ${ }^{2}$, Paul Elson ${ }^{3}$, Cristina Magi-Galluzzi ${ }^{4}$ and \\ Donna E Hansel ${ }^{1,4,5,6}$
}

${ }^{1}$ Glickman Urological and Kidney Institute, Cleveland Clinic, Cleveland, OH, USA; ${ }^{2}$ Department of Cancer Biology, Cleveland Clinic, Cleveland, OH, USA; ${ }^{3}$ Quantitative Health Sciences, Cleveland Clinic, Cleveland, OH, USA; ${ }^{4}$ Pathology and Laboratory Medicine Institute, Cleveland Clinic, Cleveland, OH, USA; ${ }^{5}$ Genomic Medicine Institute, Cleveland Clinic, Cleveland, OH, USA and ${ }^{6}$ Taussig Cancer Institute, Cleveland Clinic, Cleveland, OH, USA

Folate hydrolase (prostate-specific antigen) 1 (FH(PSA)1), also known as prostate-specific membrane antigen (PSMA), is a transmembrane receptor expressed on prostate cancer cells that correlates with a more aggressive phenotype. Recent studies have demonstrated FH(PSA)1 expression in numerous benign and malignant tissue types, as well as the malignant neovasculature. As $\mathrm{FH}(\mathrm{PSA}) 1$ represents a diagnostic immunomarker for prostate cancer, we explored its expression pattern in various subtypes of bladder cancer. Immunohistochemical analysis (IHC) of FH(PSA)1 was performed using tissue microarrays constructed from 167 bladder cancers, including 96 urothelial carcinomas (UCCs), 37 squamous cell carcinomas, 17 adenocarcinomas and 17 small cell carcinomas. We used a FH(PSA)1 monoclonal antibody obtained from Dako (clone 3E6, dilution 1:100), which recognizes the epitope present in the 57-134 amino acid region of the extracellular portion of the PSMA molecule. Intensity of IHC staining was scored as $\mathbf{0}$ (no expression) to $3+$ (strong expression), with 2-3 + IHC considered a positive result. FH(PSA)1 demonstrated expression in a subset of bladder cancers and was most common in small cell carcinoma $(3 / 17 ; 18 \%)$, with concurrent expression in non-small cell components in a subset of cases (2/6). FH(PSA)1 expression was less frequent in UCC $(3 / 96 ; 3 \%)$ and adenocarcinoma $(2 / 17 ; 12 \%)$. None of the squamous cell carcinomas demonstrated tumor cell expression of FH(PSA)1. However, all bladder cancers examined expressed FH(PSA)1 in the tumor vasculature, suggesting a potential role for this molecule in mediating new vessel ingrowth. FH(PSA)1 may occasionally be expressed in various subtypes of bladder cancer. These findings suggest cautious use of $\mathrm{FH}(\mathrm{PSA}) 1$ as a diagnostic marker for prostatic tissue invading the bladder. The finding of FH(PSA)1 in the bladder cancer neovasculature suggests that this molecule may promote tumor growth and may represent a potential new vascular target in this disease.

Modern Pathology (2011) 24, 1521-1529; doi:10.1038/modpathol.2011.112; published online 1 July 2011

Keywords: adenocarcinoma; bladder; folate hydrolase (prostate-specific antigen) 1; PSMA; small cell carcinoma; urothelial carcinoma; vasculature

In 2009, the lifetime risk for developing bladder cancer was 1 in 27 for males and 1 in 85 for females. ${ }^{1}$ Although urothelial carcinoma (UCC) comprises 90-95\% of bladder cancers in Western countries,

Correspondence: Dr DE Hansel, MD, PhD, Department of Anatomic Pathology, Cleveland Clinic, 9500 Euclid Avenue, Desk L25, Cleveland, OH 44195, USA.

E-mail: hanseld@ccf.org

Received 16 October 2010; revised 4 May 2011; accepted 4 May 2011; published online 1 July 2011 adenocarcinoma, small cell carcinoma and squamous cell carcinoma are also seen. The course of high-grade bladder cancer is often characterized by a high recurrence rate with a tendency toward progression. In poorly differentiated cases, immunohistochemical (IHC) stains may be used to rule out a prostate adenocarcinoma secondarily involving the bladder, including prostate-specific antigen (PSA), prostate-specific alkaline phosphatase (PSAP), P501S (prostein) and folate hydrolase (PSA) 1 FH(PSA)1. ${ }^{2}$ The expression of prostate-specific 
membrane antigen (PSMA) in primary bladder cancer, however, has not been examined in detail and such findings are critical in determining its value in distinguishing bladder and prostate carcinomas.

FH(PSA)1 is a transmembrane receptor classically expressed on prostate cancer cells and its expression increases progressively in higher grade cancers, metastatic disease and hormone-refractory disease. ${ }^{3-5}$ Recent studies have demonstrated variable levels of $\mathrm{FH}(\mathrm{PSA}) 1$ expression in numerous other tissue types, including cardiac tissue, pancreatic islet cells, lung adenocarcinoma, gastric cancer and colorectal cancer. ${ }^{6}$ Some studies have demonstrated FH(PSA)1 expression in the normal urothelium; however, conflicting results make the true expression pattern unclear. $^{6-9}$ Additionally, few studies have examined FH(PSA)1 expression in other bladder cancer subtypes, with only one evaluating primary bladder adenocarcinoma ${ }^{2}$ and none evaluating either squamous cell carcinoma or small cell carcinoma. Finally, FH(PSA)1 has recently been found to be strongly and reliably expressed on the malignant neovasculature of many solid neoplasms. ${ }^{6}$ Its expression in bladder cancer cells and the associated tumor vasculature has not been previously described.

Expression of FH(PSA)1 in bladder tumors has important implications for diagnostic pathology, and while its expression has been well documented in prostatic adenocarcinoma, its expression in bladder cancer subtypes is unclear. Thus, we evaluated the expression of FH(PSA) 1 in bladder cancer subtypes and the associated tumor vasculature to determine whether it may serve as a possible target in future therapeutic studies.

\section{Materials and methods}

This study was approved by the Cleveland Clinic Institutional Review Board. We utilized 167 formalin-fixed, paraffin-embedded bladder cancer cystectomy specimens obtained from the Cleveland Clinic surgical pathology archives, including 96 UCCs, 17 adenocarcinomas, 37 squamous cell carcinomas and 17 small cell carcinomas. All of these cases showed tumor invasion into the detrusor muscle. UCC cases used for study included high-grade, conventional UCC tumors without variant differentiation. Patients with precedent or concurrent high-grade prostatic adenocarcinoma, colonic adenocarcinoma or documented metastatic disease from another anatomic location were excluded from analysis. All slides were re-reviewed to confirm the final diagnosis. A retrospective review of patient records was performed to determine patient demographics, clinicopathologic features and outcomes.

Tissue microarrays (TMAs) were constructed for individual bladder cancer subtypes. Each specimen was represented by four $1.0-\mathrm{mm}$ cores on TMA to obtain adequate representation of different regions of neoplastic cells to assess for intratumoral histologic and immunophenotypic heterogeneity, which has been demonstrated to adequately represent tissue variation within UCC. ${ }^{10}$ TMAs were stained with $\mathrm{FH}(\mathrm{PSA}) 1$ using a monoclonal antibody against the 57-134 amino acid region of the extracellular portion of FH(PSA)1 (clone 3E6, 1:100, Dako, Carpinteria, CA, USA) using an automated Ventana stainer. IHC labeling was evaluated by one of the authors (DEH) and was scored semi-quantitatively as 0 , no staining; 1 , weak staining; 2 , moderate staining; and 3, intense staining. A positive result was considered an intensity of $2-3+$. For the neovasculature, the percentage of vessels with positive immunoreactivity for FH(PSA)1 was measured on $10 \times 1 \mathrm{~mm}^{2}$ fields under light microscopy. All samples were also evaluated for PSA immunoreactivity, which is commonly employed as a concurrent stain to distinguish prostatic from urothelial origin.

Categorical data were summarized as frequency counts and percentages; quantitative data were summarized as means \pm s.d., medians and ranges. Overall and failure-free survival were calculated from the date of diagnosis to the date of death, and the date of documented progression or death, whichever came first, respectively. Associations between clinicopathologic characteristics and histology and FH(PSA)1 expression in the tumor were examined using $\chi^{2}$ tests, Cochran-MantelHaenszel tests stratified by histology (for categorical variables), and the Kruskal-Wallis test and stratified (by histology) Wilcoxon rank sum test (for quantitative variables). The log-rank test and Cox proportional hazards model, stratified by histology were used for comparisons of overall and failurefree survival. All tests of statistical significance were two-sided. Data analyses were performed using SAS version 8.0 (SAS Institute, Cary, NC, USA) and StatXact 7.0 (Cytel, Cambridge, MA, USA).

\section{Results}

We evaluated 167 patients with muscle-invasive bladder cancer, including 96 UCCs, 17 adenocarcinomas, 37 squamous cell carcinomas and 17 small cell carcinomas (Table 1). Overall $75 \%$ of patients were male, median age was 71 (range 37-90), most patients had T3 tumors $(62 \%)$, and most patients had positive lymph nodes at surgery (63\%). Histologies differed with respect to age; patients with adenocarcinomas tending to be younger $(P<0.001)$.

FH(PSA)1 expression appeared most robust in small cell carcinoma, of which 3 cases out of 17 stained positively for PSMA (18\%; Figure 1). All staining was cytoplasmic in nature and ranged from $2+(n=1)$ to $3+(n=2)$, mean $2.67+$, median $3+$, in a focal to diffuse manner (Table 2). Of the 3 positive cases, all patients died from metastatic 
Table 1 Patient characteristics

\begin{tabular}{|c|c|c|c|c|c|}
\hline Factor & Adeno & Small cell & Urothelial & Squamous & $\mathrm{P}$-value \\
\hline \multicolumn{6}{|l|}{ Gender } \\
\hline Female & $6(35 \%)$ & $3(18 \%)$ & $18(19 \%)$ & $15(41 \%)$ & \\
\hline Male & $11(65 \%)$ & $14(82 \%)$ & $78(81 \%)$ & $22(59 \%)$ & $0.05^{\mathrm{a}}$ \\
\hline \multicolumn{6}{|l|}{ Age } \\
\hline Mean \pm s.d. & $59.9 \pm 12.0$ & $66.2 \pm 12.8$ & $71.8 \pm 10.7$ & $69.1 \pm 8.5$ & \\
\hline Median & 62 & 70 & 74 & 72 & $<0.001^{\mathrm{b}}$ \\
\hline Range & $37-78$ & $39-86$ & $37-90$ & $46-80$ & \\
\hline \multicolumn{6}{|l|}{ LN status } \\
\hline Negative & $3(18 \%)$ & $12(71 \%)$ & $30(31 \%)$ & $16(43 \%)$ & \\
\hline Positive & $14(82 \%)$ & $5(29 \%)$ & $66(69 \%)$ & $21(57 \%)$ & $0.001^{\mathrm{a}}$ \\
\hline \multicolumn{6}{|c|}{ Pathologic T-stage } \\
\hline 1 & - & - & $2(2 \%)$ & - & \\
\hline 2 & $2(12 \%)$ & $11(65 \%)$ & $23(24 \%)$ & $16(43 \%)$ & \\
\hline 3 & $13(76 \%)$ & 5 (29\%) & $65(68 \%)$ & $21(57 \%)$ & \\
\hline 4 & $2(12 \%)$ & $1(6 \%)$ & $6(6 \%)$ & - & $0.002^{\mathrm{b}}$ \\
\hline \multicolumn{6}{|l|}{ PSMA } \\
\hline Negative & $15(88 \%)$ & $14(82 \%)$ & $93(97 \%)$ & $37(100 \%)$ & \\
\hline Positive & $2(12 \%)$ & 3 (18\%) & $3(3 \%)$ & - & $0.03^{\mathrm{a}}$ \\
\hline
\end{tabular}

${ }^{\mathrm{a}}$ Exact $\chi^{2}$ test.

${ }^{\mathrm{b}}$ Exact Kruskal-Wallis test.
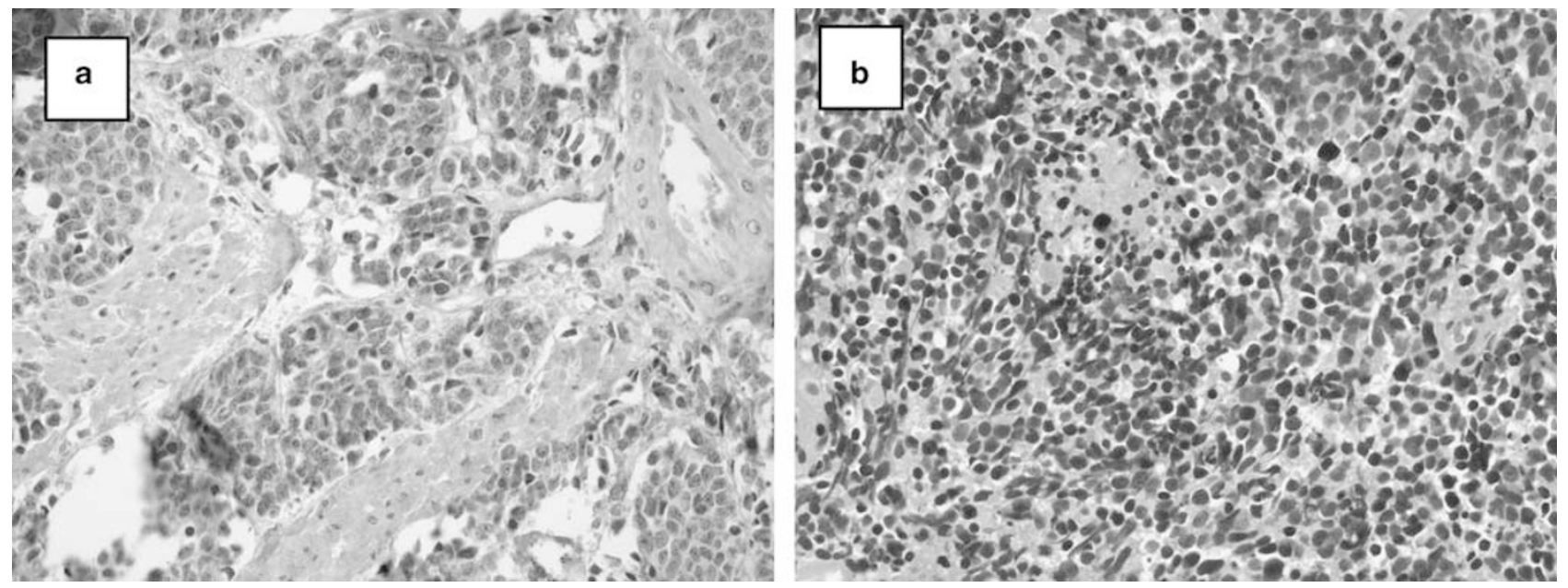

Figure 1 Folate hydrolase (prostate-specific antigen) 1 (FH(PSA)1) expression in small cell carcinoma (3/17; 18\%); staining was cytoplasmic in all cases and focal in 2 of 3. (a) Staining with a monoclonal antibody against the 57-134 amino acid region of the extracellular portion of FH(PSA)1. (b) Staining with hematoxylin and eosin demonstrates small cells with a round to fusiform shape, scant cytoplasm, with fine nuclear chromatin, absence of nucleoli and high mitotic activity.

disease at 3,5 and 12 months after surgical resection, respectively. Only one of the patients was found to have lymph node metastases. The other two patients were lymph node negative at the time of surgical resection.

Of the 17 adenocarcinomas examined, 2 demonstrated immunoreactivity for FH(PSA)1 (12\%; Figure 2). Of these, both demonstrated focal cytoplasmic staining, with an intensity of $2+$ and $3+$, respectively (Table 2). One patient subsequently developed metastatic disease, whereas the second patient was lost to follow-up. Expression in UCC and squamous cell carcinoma, in contrast, appeared less common. Only 3 out of 96 cases of UCC (3\%) demonstrated positive immunoreactivity for FH(PSA)1, with 2 patients demonstrating lymph nodes metastases at cystectomy, both of whom subsequently died of disease. Of the three cases of UCC that showed immunoreactivity for PSMA (Figure 3), all cases demonstrated $2+$ cytoplasmic 
staining, mean $2+$ and median $2+$, with one staining diffusely and two cases staining focally (Table 2). All 37 squamous cell carcinomas lacked immunoreactivity for FH(PSA)1 (0/37; 0\%).

Table 2 FH(PSA)1 staining in FH(PSA)1 expressing cases

\begin{tabular}{lcl}
\hline Cell type & Staining intensity & Location of staining \\
\hline UCC & $2+$ & Cytoplasmic \\
UCC & $2+$ & Cytoplasmic \\
UCC & $2+$ & Cytoplasmic \\
Adenocarcinoma & $2+$ & Cytoplasmic \\
Adenocarcinoma & $3+$ & Cytoplasmic \\
Small cell & $2+$ & Cytoplasmic \\
Small cell & $3+$ & Cytoplasmic \\
Small cell & $3+$ & Cytoplasmic \\
\hline
\end{tabular}

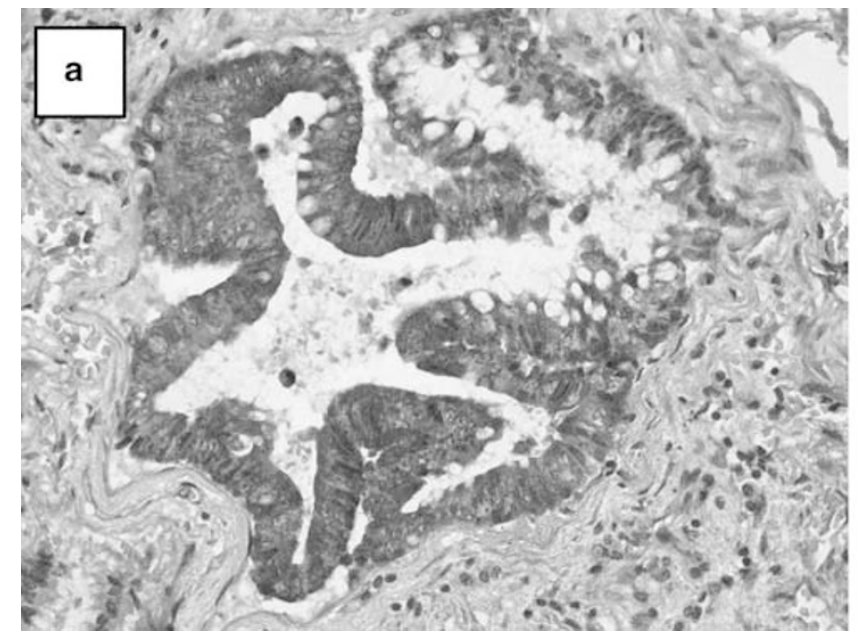

A summary of $\mathrm{FH}(\mathrm{PSA}) 1$ immunoreactive cases is presented in Table 1; overall eight patients $(6 \%)$ demonstrated FH(PSA)1 immunoreactivity. None of the samples, regardless of histology, showed immunoreactivity for PSA. FH(PSA)1 was associated with histologic subtype $(P=0.03)$; with some suggestion that FH(PSA)1 was expressed less frequently in UCCs ( $3 \%$ positive) and squamous cell carcinomas ( $0 \%$ positive) as compared with adenocarcinomas $(12 \%)$ or small cell carcinomas (18\%); $P=0.01$. In addition, $\mathrm{FH}(\mathrm{PSA}) 1$ appeared to demonstrate a trend toward expression in pathologic stage 4 tumors $(P=0.12)$ (Table 3$)$. Neither overall survival nor failure-free survival was associated with $\mathrm{FH}(\mathrm{PSA}) 1$ expression $(P=0.52$ and 0.92 , respectively), although due to the low number of FH(PSA)1-expressing patients, no definitive

Figure 2 Folate hydrolase (prostate-specific antigen) 1 (FH(PSA)1) expression in adenocarcinoma (2/17; 12\%); staining was cytoplasmic and focal in both cases. (a) Staining with a monoclonal antibody against the 57-134 amino acid region of the extracellular portion of FH(PSA)1. (b) Staining with hematoxylin and eosin demonstrates columnar epithelium in a glandular configuration, with large, dark nuclei and signet-ring cells.
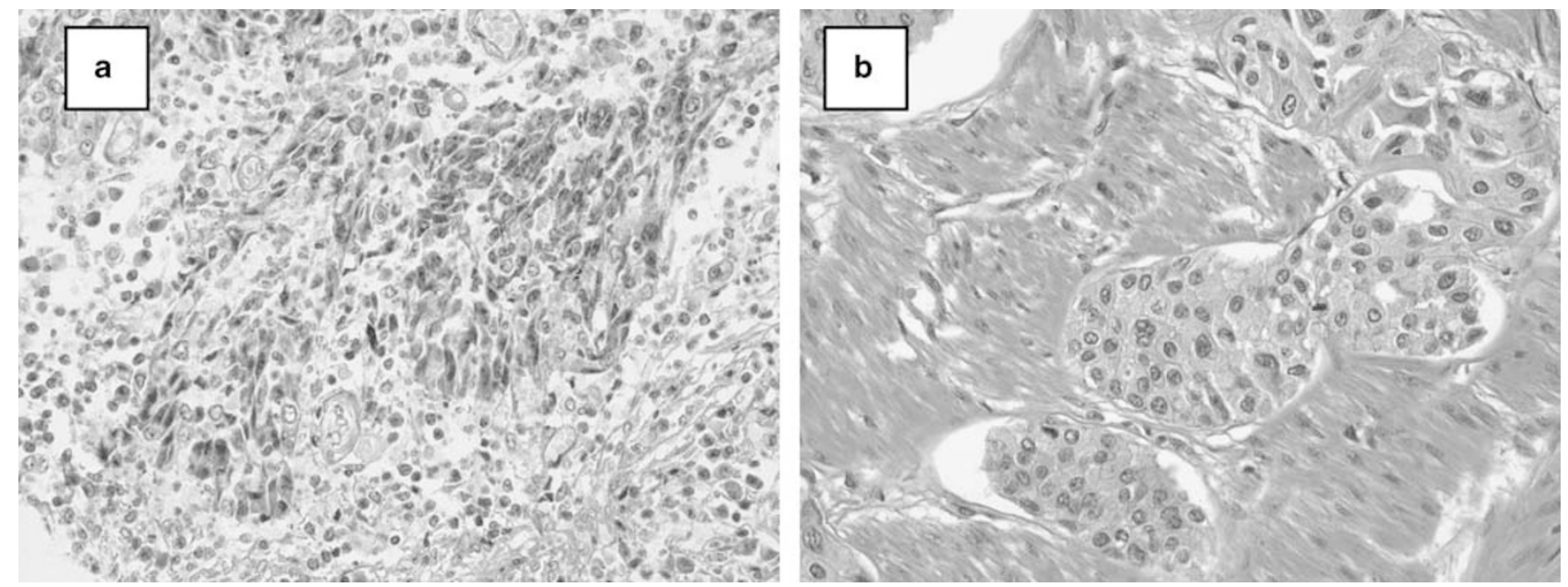

Figure 3 Folate hydrolase (prostate-specific antigen) 1 (FH(PSA)1) expression in urothelial carcinoma (3/96; 3\%); staining was cytoplasmic in all cases and focal in 2/3. (a) Staining with a monoclonal antibody against the 57-134 amino acid region of the extracellular portion of FH(PSA)1. (b) Staining with hematoxylin and eosin demonstrates large pleomorphic cells with abundant cytoplasm, irregular nuclei and increased mitotic activity. 
Table 3 Patient characteristics and FH(PSA)1 expression

\begin{tabular}{|c|c|c|c|}
\hline Factor & Negative & Positive & $\mathrm{P}$-value ${ }^{\mathrm{a}}$ \\
\hline \multicolumn{4}{|l|}{ Gender } \\
\hline Female & $41(98 \%)$ & $1(2 \%)$ & \\
\hline Male & $118(94 \%)$ & $7(6 \%)$ & 0.66 \\
\hline \multicolumn{4}{|l|}{ Age } \\
\hline Mean \pm s.d. & $69.8 \pm 10.6$ & $62.6 \pm 18.8$ & \\
\hline $\begin{array}{l}\text { Median } \\
\text { Range }\end{array}$ & 72 & $\begin{array}{c}60 \\
30-8\end{array}$ & $0.77^{\mathrm{b}}$ \\
\hline & & & \\
\hline \multicolumn{4}{|l|}{ LN status } \\
\hline Negative & $59(97 \%)$ & $2(3 \%)$ & \\
\hline Positive & $100(94 \%)$ & $6(6 \%)$ & $0.41^{\mathrm{a}}$ \\
\hline \multicolumn{4}{|l|}{ Pathologic T-stage } \\
\hline 1 & $2(100 \%)$ & - & \\
\hline 2 & $50(96 \%)$ & $2(4 \%)$ & \\
\hline 3 & $100(96 \%)$ & $4(4 \%)$ & \\
\hline 4 & $7(78 \%)$ & $2(22 \%)$ & $0.19,0.12$ \\
\hline \multicolumn{4}{|l|}{ Cell type } \\
\hline Adenocarcinoma & $15(88 \%)$ & $2(12 \%)$ & \\
\hline Small cell & $14(82 \%)$ & $3(18 \%)$ & \\
\hline Urothelial & $93(97 \%)$ & $3(3 \%)$ & \\
\hline Squamous cell & $37(100 \%)$ & - & $0.03^{\mathrm{c}}$ \\
\hline
\end{tabular}

${ }^{a}$ Exact Cochran-Mantel-Haenszel test, stratified by histologic subtype unless otherwise noted.

${ }^{\mathrm{b}}$ Exact Wilcoxon rank sum test, stratified by histologic subtype.

${ }^{\mathrm{C}}$ Exact $\chi^{2}$ test. conclusion can be drawn regarding the association between FH(PSA)1 and outcomes (Table 4).

We next evaluated the associated tumor vasculature for FH(PSA)1. All tumors examined, regardless of subtype, demonstrated staining for FH(PSA)1 on associated blood vessels (Figure 4). Staining ranged from $1+$ to $2-3+$, and ranged from 10 to $100 \%$ per specimen (Table 5). Small cell carcinoma demonstrated the highest percentage of vessels staining positively for FH(PSA)1 (mean $48.53 \%$, range 10$90 \%$ ), followed by squamous cell carcinoma (mean $21.52 \%$, range $10-45 \%$ ), UCC (mean $21.09 \%$, range $10-60 \%$ ) and adenocarcinoma (mean $16.47 \%$, range $10-40 \%$ ). This same trend was followed for mean vessel staining intensity, where small cell carcinoma stained the strongest $(2.65+)$, followed by squamous cell carcinoma $(1.86+)$, UCC $(1.67+)$ and adenocarcinoma $(1.53+)$. FH(PSA)1 expression was not evident in adjacent, non-neoplastic regions of the bladder wall. No definitive relationship between FH(PSA)1 expression in the tumor cells and the adjacent neovasculature was identified.

\section{Discussion}

Bladder cancer occurs as several different histopathologic subtypes, with the major subcategories

Table 4 Failure-free and overall survival (in months)

\begin{tabular}{|c|c|c|c|c|c|c|}
\hline \multirow[t]{2}{*}{ Factor } & \multicolumn{3}{|c|}{$\begin{array}{c}\text { Failure-free survival }(\mathrm{n}=156) \\
\text { No. }(\%)\end{array}$} & \multicolumn{3}{|c|}{$\begin{array}{c}\text { Overall survival }(\mathrm{n}=157) \\
\text { No. }(\%)\end{array}$} \\
\hline & Failures & Median & $\mathrm{P}$-value ${ }^{\mathrm{a}}$ & Deaths & Median & $\mathrm{P}$-value \\
\hline \multicolumn{7}{|l|}{$F H(P S A) 1$} \\
\hline Negative & $94(64 \%)$ & 18.0 & & $88(59 \%)$ & 22.0 & \\
\hline Positive & $7(88 \%)$ & 12.5 & 0.52 & $5(62 \%)$ & 25.5 & 0.92 \\
\hline \multicolumn{7}{|l|}{ Gender } \\
\hline Female & $23(62 \%)$ & 15.0 & & $18(47 \%)$ & 23.0 & \\
\hline Male & $78(67 \%)$ & 18.0 & 0.88 & $75(63 \%)$ & 22.0 & 0.74 \\
\hline Age in decades & - & - & $0.007^{\mathrm{b}}$ & - & - & $0.003^{\mathrm{b}}$ \\
\hline \multicolumn{7}{|l|}{ LN status } \\
\hline Negative & $27(48 \%)$ & 38.0 & & $27(47 \%)$ & 38.0 & \\
\hline Positive & $74(74 \%)$ & 15.0 & 0.005 & $66(66 \%)$ & 21.0 & 0.02 \\
\hline \multicolumn{7}{|l|}{ Pathologic T-stage } \\
\hline 1 & $1(50 \%)$ & NA & & $1(50 \%)$ & NA & \\
\hline 2 & $24(50 \%)$ & 38.0 & & $24(49 \%)$ & 38.0 & \\
\hline 3 & $68(70 \%)$ & 16.0 & & $62(64 \%)$ & 21.0 & \\
\hline 4 & $8(89 \%)$ & 12.0 & $0.02^{\mathrm{b}}$ & $6(67 \%)$ & 14.0 & $0.05^{\mathrm{b}}$ \\
\hline \multicolumn{7}{|l|}{ Cell type } \\
\hline Adeno & $15(94 \%)$ & 9.5 & & $9(56 \%)$ & 51.0 & \\
\hline Small cell & $12(71 \%)$ & 11.0 & & $12(71 \%)$ & 12.0 & \\
\hline Urothelial & $57(60 \%)$ & 18.0 & & $57(61 \%)$ & 21.0 & \\
\hline Squamous cell & $17(61 \%)$ & 32.0 & $0.19^{\mathrm{c}}$ & $15(54 \%)$ & 32.0 & $0.68^{\mathrm{c}}$ \\
\hline
\end{tabular}

${ }^{\mathrm{a}}$ Exact log-rank test stratified by histologic subtype unless otherwise noted.

${ }^{\mathrm{b}}$ Wald test from proportional hazards model stratified by histologic subtype.

${ }^{\mathrm{c}}$ Exact log-rank test (unstratified). 

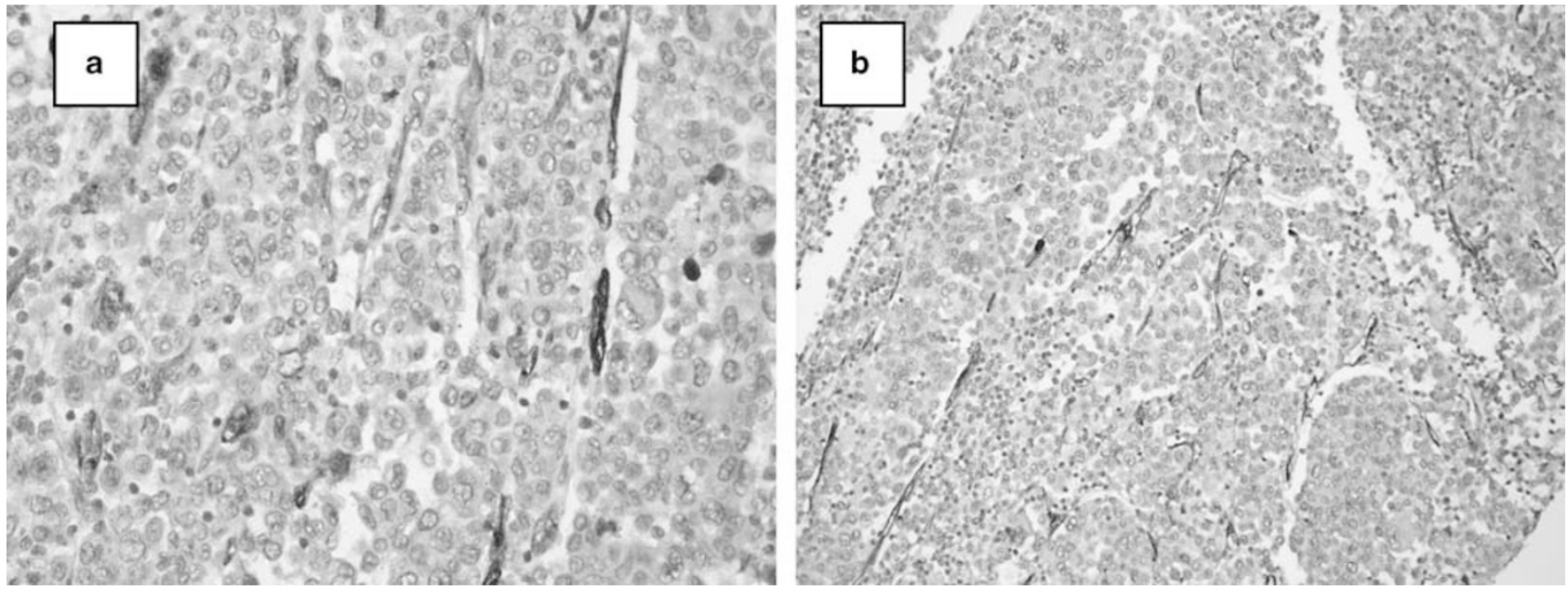

Figure 4 (a) Folate hydrolase (prostate-specific antigen) 1 (FH(PSA)1) expression in the tumor vasculature. All tumors demonstrated staining $(168 / 168,100 \%)$. Staining ranged from $1+$ to $2-3+$, and ranged from 10 to $100 \%$ per specimen. (b) A second example of FH(PSA)1 staining on the malignant neovasculature.

Table 5 Percentage and intensity of blood vessels staining positively for $\mathrm{FH}(\mathrm{PSA}) 1$

\begin{tabular}{lccc}
\hline Histology & $\begin{array}{c}\text { No. of cases } \\
\text { staining positively } \\
\text { for FH(PSA)1 }\end{array}$ & $\begin{array}{c}\text { Mean vessel } \\
\text { staining (\%) }\end{array}$ & $\begin{array}{c}\text { Mean } \\
\text { staining } \\
\text { intensity }\end{array}$ \\
\hline UCC & 96 & 21.09 & $1.67+$ \\
AdenoCA & 17 & 16.47 & $1.53+$ \\
Squamous & 36 & 21.52 & $1.86+$ \\
Small cell & 17 & 48.53 & $2.65+$ \\
\hline
\end{tabular}

including UCC, squamous cell carcinoma, adenocarcinoma and small cell carcinoma. Although often readily diagnosed on $\mathrm{H} \& \mathrm{E}$, occasional cases present diagnostic challenges because of poorly differentiated morphology or concern over secondary spread from an external site. In such cases, IHC analysis is often employed to exclude metastatic or direct spread of a carcinoma into the bladder wall. One of the most common secondary cancers involving the bladder is prostatic adenocarcinoma, which is typically glandular in appearance but may mimic UCC in high-grade tumors or may rarely demonstrate small cell differentiation. To address this possibility, IHC stains for common prostate markers are often used, including PSA, PSAP, P501S (prostein) and FH(PSA)1. ${ }^{2,11}$ The benign urothelium has traditionally lacked immunoreactivity for PSA and PSAP, as has UCC. ${ }^{12}$ However, the ability of highgrade carcinomas to aberrantly express markers not unique to their location is well recognized and may be problematic in difficult cases. The goal of this paper was to evaluate the expression of FH(PSA) 1 in various forms of bladder cancer to determine its utility in excluding a prostatic primary carcinoma.

FH(PSA)1 (FH 1, glutamate carboxypeptidase II) is a $120-\mathrm{kDa}$ type II transmembrane glycoprotein first characterized in prostate cancer. ${ }^{3,10,13}$ Under normal conditions, FH(PSA)1 is expressed in a multitude of tissues, including prostatic epithelium, duodenal brush border epithelium, proximal renal tubular epithelium, colonic ganglionic cells and benign breast epithelium. ${ }^{6,7}$ FH(PSA) 1 is also overexpressed in prostatic adenocarcinoma and its associated metastases. ${ }^{14-16}$ In this group, its expression correlates directly with a higher tumor grade, stage and adverse clinical outcomes, as measured using reverse transcription-PCR ${ }^{17}$ and IHC staining for FH(PSA)1 using a 7E11 antibody. ${ }^{14}$ Other tumor types that have been shown to express FH(PSA)1 include kidney, stomach, small intestine, colon, lung, adrenal and testis. ${ }^{6,18}$ Currently, the exact function of FH(PSA)1 is unknown, although it is known to have $\mathrm{FH}$ and naladase activity in cells of the intestinal brush border, brain and spinal cord. ${ }^{6} \mathrm{FH}$ within the intestinal brush border hydrolyzes dietary polyglutamylated folate to folate, thereby enhancing folate uptake. ${ }^{17}$ In the brain it hydrolyzes $N$-acetyl-L-aspartyl-L-glutamate to the more potent neurotransmitter glutamate. ${ }^{19}$ FH(PSA)1 may be localized to cytoplasmic or membranous domains, and the implications of this localization are still unclear. Recent studies suggest that there may be a unique mechanism for proteolytic cleavage with intracellular transport of the fully glycosidated external portion of the extracellular domain that is seen in some high-grade glioblastomas. ${ }^{20}$ Neither the proteolytic site nor transport system have been described for either gliobastomas or other tumors. ${ }^{20}$ Thus, the role of FH(PSA) 1 in oncogenic conditions and the neovasculature is still being elucidated.

FH(PSA)1 has been recently shown to be expressed in new vessels arising in reparative conditions, ${ }^{21}$ suggesting that it may function in regenerative angiogenesis by increasing the local 
availability of folate. ${ }^{21}$ Folate can increase the levels of the proangiogenic molecule nitric oxide (NO) by regenerating the endothelial NO synthase cofactor, tetrahydrobiopterin $\left(\mathrm{BH}_{4}\right){ }^{22}$ Studies in knockout mice in which the mouse ortholog of FH(PSA)1 was eliminated, the mice had a dramatically reduced ability to undergo angiogenesis of a subcutaneously introduced Matrigel plug. Endothelial cells derived from the knockout mice had less ability for $\beta_{1}$ integrin activation. ${ }^{23}$

Expansion of this investigative line has also identified FH(PSA)1 expression in tumor neovasculature at numerous sites. Specifically, FH(PSA)1 is strongly expressed in the malignant neovasculature of clear cell renal carcinoma, testicular embryonal cell carcinoma, colonic adenocarcinoma, neuroendocrine carcinoma, glioblastoma multiforme, malignant melanoma, pancreatic ductal carcinoma, non-small cell lung carcinoma and breast carcinoma. As FH(PSA)1 is membrane bound and has both intracellular and extracellular domains, its localization to both tumor cells and associated vessels raises the possibility of targeting this molecule for therapeutic intervention. This possibility is further highlighted by the lack of FH(PSA)1 expression by normal endothelium under non-reactive conditions. ${ }^{8,18,24,25}$ As an example, antibodies to FH(PSA)1 have been used for in vivo localization and treatment of metastatic prostate cancer ${ }^{15,16}$ and have been shown to target the neovasculature endothelium of multiple solid tumor malignancies in humans. ${ }^{26,27}$

Review of the literature reveals conflicting results for the expression of FH(PSA)1 in normal urothelium and UCC (Table 6). In normal urothelium, reported $\mathrm{FH}(\mathrm{PSA}) 1$ expression ranges from ubiquitous $\left(18 / 18\right.$ specimens $^{6}$ and $9 / 9$ specimens $\left.^{9}\right)$ to non-existent $\left(0 / 846^{7}\right.$ and $0 / 5$ specimens $\left.^{8}\right)$. In UCC specimens, FH(PSA)1 has been reported to exhibit a striking range of expression as well, present in 52/52 specimens, ${ }^{9} 18 / 21,{ }^{6} 59 / 346^{7}$ and $0 / 5$ specimens. ${ }^{8}$
These varied results likely stem from the ability of early anti-FH(PSA)1 antibodies to stain only frozen tissue rather than formalin-fixed, paraffinembedded tissues. In addition, previous antibodies have a range of sensitivity and specificity in FH(PSA)1 detection. The 3E6 monoclonal antibody against the extracellular domain, which we used for our study, is the only commercially available antibody that has been validated for use in both frozen and paraffin-embedded tissue, with minimal artifact. $^{18}$

Using the 3E6 antibody, we evaluated the major subtypes of bladder cancer. In general, few bladder cancers expressed FH(PSA)1, with small cell carcinoma and primary bladder adenocarcinoma representing the most commonly expressing subtypes (18\% and $12 \%$, respectively). The co-expression of FH(PSA)1 on admixed forms of bladder cancer present in small cell carcinoma suggests the possibility that upregulation of FH(PSA)1 may occur early in the course of disease. Only one previous study has examined FH(PSA)1 expression in bladder adenocarcinoma using the 3E6 antibody ${ }^{2}$ and identified expression in 27\% (10/37) cases, which perhaps reflects an underlying propensity of expression in glandular-type tissue. Only $3 \%$ of UCC specimens demonstrated FH(PSA)1 expression and no case of squamous cell carcinoma demonstrated expression.

Outcome assessment in our study indicated that after adjusting for histologic subtype, decreased failure-free and overall survival were associated with positive lymph node status $(P=0.005$ and 0.02 , respectively), higher pathologic stage $(P=0.02$ and 0.05 , respectively) and age $(P=0.007$ and 0.003 , respectively). Neither outcome, however, was associated with FH(PSA)1 expression after adjusting for histology ( $P=0.52$ and 0.92 , respectively). This result held true even after adjusting for lymph node status, pathologic stage or age. These findings

Table 6 Literature review of FH(PSA)1 expression on normal urothelium and bladder carcinoma

\begin{tabular}{|c|c|c|c|c|c|c|c|}
\hline Author & $\begin{array}{l}\text { Normal } \\
\text { urothelium, } \\
\mathrm{N}(\%)\end{array}$ & $\begin{array}{l}U C C, \\
\mathrm{~N}(\%)\end{array}$ & $\begin{array}{c}\text { Malignant } \\
\text { neovasculature, } \\
\mathrm{N}(\%)\end{array}$ & $\begin{array}{c}\text { UCC derived } \\
\text { cell lines, } \\
\text { N (\%) }\end{array}$ & $\begin{array}{l}\text { AdenoCA, } \\
\text { N (\%) }\end{array}$ & $\begin{array}{c}\text { Squamous } \\
\text { cell CA, } \\
\text { N }(\%)\end{array}$ & $\begin{array}{l}\text { Monoclonal } A b \text {, } \\
\mathrm{N}(\%)\end{array}$ \\
\hline Lane et $a l^{2}$ & & & & & $10 / 37(27.03 \%)$ & & $3 \mathrm{E} 6$ \\
\hline Kinoshita et $a l^{6}$ & 18/18 (100\%) & $18 / 21(85.71 \%)$ & & & & & $\begin{array}{l}\text { 24.4E6 } \\
\text { 7E11.C5 }\end{array}$ \\
\hline $\begin{array}{l}\text { Mhawech- } \\
\text { Fauceglia et } a l^{7}\end{array}$ & $0 / 846(0 \%)$ & $59 / 346(17.05 \%)$ & & & $1 / 5(20 \%)$ & $1 / 8(12.5 \%)$ & Y-PSMA1 \\
\hline Chang et $a l^{8}$ & $0 / 5(0 \%)$ & $0 / 5(0 \%)$ & $5 / 5(100 \%)$ & & & & $\begin{array}{l}\text { 7E11 } \\
\text { J591 } \\
\text { J415 } \\
\text { PEQ 226.5 } \\
\text { PM2J004.5 }\end{array}$ \\
\hline Gala et $a l^{9}$ & 9/9 (100\%) & $52 / 52(100 \%)$ & & $2 / 3(66.67 \%)$ & & & CYT-351 \\
\hline Milowsky et $a l^{26}$ & & & $3 / 3(100 \%)$ & & & & J591 \\
\hline
\end{tabular}


suggest that the primary determinants of survival are lymph node status and pathologic stage, being more clinically relevant than the histologic subtype or FH(PSA)1 expression pattern. Although this study is not adequately powered to definitively address the relationship between $\mathrm{FH}(\mathrm{PSA}) 1$ positivity and outcome, future studies on a larger number of cases would be warranted to determine whether FH(PSA)1 expression is a driver of outcomes.

No previous studies specifically have looked at the relationship between patient characteristics, such as age $(P=0.77)$, gender $(P=0.66)$, and lymph nodal status $(P=0.41)$, to FH(PSA)1 expression. Our analysis revealed that $\mathrm{FH}(\mathrm{PSA}) 1$ expression was only correlated with tumor histology $(P=0.03)$, and no other patient characteristic, with the possible exception of pathologic stage. Given its postulated role with respect to angiogenesis and malignancy, such correlations would not be expected.

In addition to expression on tumor cells, FH(PSA)1 is also frequently expressed on tumor vasculature and has been reported in small series of UCC specimens. ${ }^{8}$ Our results are consistent with these reports, as we have identified expression of FH(PSA) 1 in the tumor vasculature of all 168 specimens. As such, FH(PSA)1 represents a unique antigen with a potential role in mediating new vessel ingrowth. As FH(PSA)1 is not expressed on the non-neoplastic vasculature, its selective expression on the malignant and regenerative neovasculature has promising therapeutic implications. Early studies utilizing the monoclonal antibody J591 to target FH(PSA)1 in patients with metastatic solid tumors have been successfully localized to the malignant neovasculature, even to distant parts of the body such as the brain. ${ }^{26}$ Future research on the utility of similar antibodies in bladder cancer is warranted given the broad distribution of this protein in bladder tumors and the malignant neovasculature.

FH(PSA)1 may be occasionally expressed in various subtypes of bladder cancer, including UCC, adenocarcinoma and small cell carcinoma. As such, the use of $\mathrm{FH}(\mathrm{PSA}) 1$ as a distinguishing tool between bladder and prostatic carcinoma should be used with caution. In addition to its expression in some bladder cancers, $\mathrm{FH}(\mathrm{PSA}) 1$ is highly expressed in bladder cancer neovasculature and may have an active role in new vessel ingrowth. However, the primary determinants of survival continue to be lymph node status and pathologic stage, being more clinically relevant than the histologic subtype or FH(PSA)1 expression pattern. Future investigation into the function of $\mathrm{FH}(\mathrm{PSA}) 1$ at this location may provide a powerful new tool in the understanding of vascular growth and tumorigenesis at this location.

\section{Disclosure/conflict of interest}

The authors declare no conflict of interest.

\section{References}

1 American Cancer Society. Bladder Cancer Overview [homepage on the Internet]. American Cancer Society; [updated 1 July 2010; cited 31 August 2010]. Available from: http://www.cancer.org/cancer/bladdercancer/ overviewguide/bladder-cancer-overview-key-statistics.

2 Lane Z, Hansel DE, Epstein JI. Immunohistochemical expression of prostatic antigens in adenocarcinoma and villous adenoma of the urinary bladder. Am J Surg Pathol 2008;32:1322-1326.

3 Israeli RS, Powell CT, Fair WR, et al. Molecular cloning of a complementary DNA encoding a prostate-specific membrane antigen. Cancer Res 1993;53: 227-230.

4 Bostwick DG, Pacelli A, Blute M, et al. Prostate specific membrane antigen expression in prostatic intraepithelial neoplasia and adenocarcinoma: a study of 184 cases. Cancer 1998;82:2256-2261.

5 Sweat SD, Pacelli A, Murphy GP, et al. Prostatespecific membrane antigen expression is greatest in prostate adenocarcinoma and lymph node metastases. Urology 1998;52:637-640.

6 Kinoshita Y, Kuratsukuri K, Landas S, et al. Expression of prostate-specific membrane antigen in normal and malignant human tissues. World J Surg 2006;30: 628-636.

7 Mhawech-Fauceglia P, Fauceglia P, Zhang S, et al. Prostate-specific membrane antigen (PSMA) protein expression in normal and neoplastic tissues and its sensitivity and specificity in prostate adenocarcinoma: an immunohistochemical study using mutiple tumour tissue microarray technique. Histopathology 2007;50: 472-483.

8 Chang SS, Reuter VE, Heston WD, et al. Five different anti-prostate-specific membrane antigen (PSMA) antibodies confirm PSMA expression in tumor-associated neovasculature. Cancer Res 1999;59:3192-3198.

9 Gala JL, Loric S, Guiot Y, et al. Expression of prostatespecific membrane antigen in transitional cell carcinoma of the bladder: prognostic value? Clin Cancer Res 2000;6:4049-4054.

10 Israeli RS, Powell CT, Corr CT, et al. Expression of the prostate-specific membrane antigen. Cancer Res 1994;54:1807-1811.

11 Chuang AY, DeMarzo AM, Veltri RW, et al. Immunohistochemical differentiation of high-grade prostate carcinoma from urothelial carcinoma. Am J Surg Pathol 2007;31:1246-1255.

12 Epstein JI. PSA and PAP as immunohistochemical markers in prostate cancer. Urol Clin North Am 1993;20:757-770.

13 Horoszewicz JS, Kawinski E, Murphy GP. Monoclonal antibodies to a new antigenic marker in epithelial prostatic cells and serum of prostatic cancer patients. Anticancer Res 1987;7:927-935.

14 Ross JS, Sheehan CE, Fisher HA, et al. Correlation of primary tumor prostate-specific membrane antigen expression with disease recurrence in prostate cancer. Clin Cancer Res 2003;9:6357-6362.

15 Bander NH, Milowsky MI, Nanus DM, et al. Phase I trial of 177lutetium-labeled J591, a monoclonal antibody to prostate-specific membrane antigen, in patients with androgen-independent prostate cancer. J Clin Oncol 2005;23:4591-4601.

16 Henry MD, Wen S, Silva MD, et al. A prostate-specific membrane antigen-targeted monoclonal antibody- 
chemotherapeutic conjugate designed for the treatment of prostate cancer. Cancer Res 2004;64: 7995-8001.

17 Carter RE, Feldman AR, Coyle JT. Prostate-specific membrane antigen is a hydrolase with substrate and pharmacologic characteristics of a neuropeptidase. Proc Natl Acad Sci USA 1996;93:749-753.

18 Haffner MC, Kronberger IE, Ross JS, et al. Prostatespecific membrane antigen expression in the neovasculature of gastric and colorectal cancers. Hum Pathol 2009;40:1754-1761.

19 Luthi-Carter R, Barczak AK, Speno H, et al. Molecular characterization of human brain N-acetylated alphalinked acidic dipeptidase (NAALADase). J Pharmacol Exp Ther 1998;286:1020-1025.

20 Mlcochova P, Barinka C, Tykvart J, et al. Prostatespecific membrane antigen and its truncated form PSM'. Prostate 2009;69:471-479.

21 Gordon IO, Tretiakova MS, Noffsinger AE, et al. Prostate-specific membrane antigen expression in regeneration and repair. Mod Pathol 2008;21: 1421-1427.
22 Stroes ES, van Faassen EE, Yo M, et al. Folic acid reverts dysfunction of endothelial nitric oxide synthase. Circ Res 2000;86:1129-1134.

23 Conway RE, Petrovic N, Li Z, et al. Prostate-specific membrane antigen regulates angiogenesis by modulating integrin signal transduction. Mol Cell Biol 2006;26: 5310-5324.

24 Liu H, Moy P, Kim S, et al. Monoclonal antibodies to the extracellular domain of prostate-specific membrane antigen also react with tumor vascular endothelium. Cancer Res 1997;57:3629-3634.

25 Chang SS, O’Keefe DS, Bacich DJ, et al. Prostate-specific membrane antigen is produced in tumor-associated neovasculature. Clin Cancer Res 1999;5:2674-2681.

26 Milowsky MI, Nanus DM, Kostakoglu L, et al. Vascular targeted therapy with anti-prostate-specific membrane antigen monoclonal antibody J591 in advanced solid tumors. J Clin Oncol 2007;25:540-547.

27 Morris MJ, Pandit-Taskar N, Divgi CR, et al. Phase I evaluation of J591 as a vascular targeting agent in progressive solid tumors. Clin Cancer Res 2007;13: 2707-2713. 